\title{
Spin-resolved correlation kinetic energy of the spin-polarized electron gas
}

\author{
John F. Dobson, ${ }^{1}$ Hung M. Le, ${ }^{1}$ and Giovanni Vignale ${ }^{2}$ \\ ${ }^{1}$ School of Science, Griffith University, Nathan, Queensland 4111, Australia \\ ${ }^{2}$ Department of Physics, University of Missouri, Columbia, Missouri 65211, USA \\ (Received 17 June 2004; revised manuscript received 17 September 2004; published 23 November 2004)
}

\begin{abstract}
We give an exact formula, based on differentiation with respect to the electron mass, for the spin resolution of the kinetic energy (KE) of a general many-electron system, including the correlation KE. We evaluate the spin-resolved correlation KE for a uniform three dimensional electron gas of arbitrary spin polarization at metallic densities, using the Singwi-Tosi-Land-Sjolander formalism. We give a very accurate scaling relation for the data thus obtained. Our results are expected to be relevant in the analysis of spintronic systems.
\end{abstract}

DOI: 10.1103/PhysRevB.70.205126

PACS number(s): 71.15.Mb, 71.10.Ca, 85.75.-d

\section{INTRODUCTION}

The energetics of the uniform interacting electron liquid is a classic problem of many-body theory, which plays an important role in many areas of condensed matter physics. Back in the 1950s the random phase approximation (RPA) was introduced to cure the divergence that appears in a naive perturbative calculation of the ground-state energy to second order in the strength of the Coulomb interaction, ${ }^{1,2}$ and an unphysical singularity in the low-temperature heat capacity of simple metals. ${ }^{3}$ Nowadays an accurate knowledge of the exchange-correlation energy-obtained through sophisticated Monte Carlo calculation ${ }^{4-7}$-is a prerequisite for the construction of approximate exchange-correlation potentials, which are used in density functional calculations ${ }^{8-10}$ of the electronic structure of systems ranging from simple atoms to macromolecules. In another application, the kinetic and the potential energy of the uniform electron liquid are used to determine, via the compressibility sum rule and the thirdmoment sum rule, the low- and high-frequency behaviors of the density-density and current-current response functions. ${ }^{11}$

Although the theory of the energetics of the electron liquid might be considered a "mature" subject, there is one question to which little attention has been paid until very recently, namely, how are the kinetic and the potential energy distributed between the two spin orientations in a spinpolarized electron liquid?

The interest in this question arises in the first place from the fact that there is a growing need to apply the spin density functional theory to spin-polarized systems, in which the distribution of the energy between the two spin components is obviously uneven (the recently discovered ferromagnetic semiconductors being a prime example). ${ }^{12-14}$ In addition, there are certain generalizations of density functional theory which do require a knowledge of the average kinetic energy of up- and down-spin electrons separately. For example, in a recent paper Qian and Vignale ${ }^{15}$ have proposed a macroscopic equation of motion for the collective dynamics of the magnetization in an itinerant-electron ferromagnet: the leading correction to the standard Landau-Lifshitz equation ${ }^{16}$ involves precisely the difference between the kinetic energies of up- and down-spin electrons, evaluated at the local densities. Knowledge of the spin-resolved kinetic energy is also required for the calculation of the high-frequency limits of the spin components of the density-density response functions, ${ }^{17}$ which in turn are used for the construction of approximate exchange-correlation potentials in the recently formulated spin-current density functional theory. ${ }^{18,19}$ In general, the spin resolution of the ground-state energy and thermodynamic functions appears naturally in theories of dynamics and transport in spin-polarized systems.

The spin resolution of the average potential energy has recently been computed by Gori-Giorgi and Perdew, ${ }^{20}$ making use of the formula that relates the average potential energy of interaction between electrons of spin $s$ and $s^{\prime}, u_{s s^{\prime}}$, to the corresponding spin-resolved pair correlation function $g_{s s^{\prime}}(r)$ :

$$
u_{s s^{\prime}}=\frac{1}{2} n_{s} n_{s^{\prime}} \int d \vec{r} \int d \vec{r}^{\prime} \frac{e^{2}}{\left|\vec{r}-\vec{r}^{\prime}\right|}\left[g_{s s^{\prime}}(\vec{r})-1\right]
$$

The pair correlation functions $g_{s s^{\prime}}(\vec{r})$ were obtained from a quantum Monte Carlo (QMC) calculation.

Unfortunately, this approach cannot be generalized immediately to determine the spin components, $t_{\uparrow}$ and $t_{\downarrow}$, of the kinetic energy. Ordinarily, one calculates the kinetic energy by first doing an integral of the potential energy over the coupling constant from zero to the physical value, and then subtracting the potential energy evaluated at the physical coupling constant. But this method does not yield $t_{\uparrow}$ and $t_{\downarrow}$ separately, because one does not know how each of the three components of the potential energy $\left(u_{\uparrow \uparrow}, u_{\downarrow \downarrow}\right.$, and $\left.u_{\uparrow \downarrow}\right)$ when integrated over the coupling constant, contributes to the two components of the kinetic energy.

In this paper we introduce a simple trick to bypass this difficulty; namely we note that if we assign two slightly different masses to up- and down-spin electrons, then the spin resolution of the kinetic energy can be rigorously obtained from the difference between the exact ground-state energy of this fictitious system and that of the physical one.

Presumably the most accurate way to obtain these energies is through QMC calculations. Although QMC calculations can certainly be performed for mixtures of species with differing masses, ${ }^{21}$ to our knowledge the detailed data required for the uniform spin polarized electron gas are not currently available. 
Therefore, we resort to a different method for the calculation of the ground-state energy, namely, the Singwi-TosiLand-Sjolander (STLS) method, ${ }^{22}$ which was originally developed for the electron liquid at metallic densities, and is known to yield very accurate values for the ground-state energy of one- and two-component systems, such as electronhole liquids. ${ }^{23}$ We will see that this method works very well also for the problem at hand.

Our calculation reveals an interesting scaling relation, whereby the spin-asymmetry of the correlation kinetic energy $\left(t_{c \uparrow}-t_{c \downarrow}\right) /\left(t_{c \uparrow}+t_{c \downarrow}\right)$ is found to be a function of the degree of spin polarization only, and not of the dimensionless Wigner-Seitz radius $r_{s}$. This suggests that one may combine the best available values of the correlation kinetic energy, obtained by QMC, with the spin asymmetry of this quantity, computed by STLS, to achieve the desired spin resolution.

The numerical results obtained in this manner should prove very useful in the theoretical analysis of spin dynamics and spin transport in electronic devices, particularly the ones based on ferromagnetic semiconductors, for which the electron liquid-based spin-density functional theory is likely to be most accurate.

\section{SPIN-RESOLVED KINETIC ENERGY VIA DIFFERENTIATION ON MASS: GENERAL MANY- ELECTRON SYSTEM}

Consider a modified many-electron system with different masses $m_{\uparrow}=\alpha_{\uparrow} m$ and $m_{\downarrow}=\alpha_{\downarrow} m$ for electrons of spin up and down, respectively, where $m$ is the true electron mass. The hamiltonian is $\hat{H}=\hat{T}+\hat{V}+\hat{U}$ where the kinetic energy operator is

$$
\begin{aligned}
\hat{T}= & -\frac{\hbar^{2}}{2 m} \int\left\{\alpha_{\uparrow}^{-1} \hat{\psi}_{\uparrow}^{\dagger}(\vec{r})\left[\vec{\nabla}-i \hbar^{-1} e \vec{A}(\vec{r})\right]^{2} \hat{\psi}_{\uparrow}(\vec{r})\right. \\
& \left.+\alpha_{\downarrow}^{-1} \hat{\psi}_{\downarrow}^{\dagger}(\vec{r})\left[\vec{\nabla}-i \hbar^{-1} e \vec{A}(\vec{r})\right]^{2} \hat{\psi}_{\downarrow}(\vec{r})\right\} d \vec{r} \\
= & \alpha_{\uparrow}^{-1} \hat{T}_{\uparrow}+\alpha_{\downarrow}^{-1} \hat{T}_{\downarrow} .
\end{aligned}
$$

$\vec{A}(\vec{r})$ is the external vector potential and $\hat{V}$ is the remaining scalar interaction with the external world (e.g., with a uniform positive background, in the special case of a homogeneous electron gas). $\hat{U}$ is the usual spin-independent bare coulomb electron-electron interaction. $\hat{V}$ can depend on position and also on spin (e.g., to represent the Zeeman effects of an external $B$ field), but must not depend on electron mass. The many-electron groundstate with fixed external potential $\hat{V}$ will be denoted $\left|\alpha_{\uparrow}, \alpha_{\downarrow}\right\rangle$, or simply |\rangle . The energy of this groundstate is

$$
E\left(\alpha_{\uparrow}, \alpha_{\downarrow}\right)=\left\langle\left|\alpha_{\uparrow}^{-1} \hat{T}_{\uparrow}+\alpha_{\downarrow}^{-1} \hat{T}_{\downarrow}+\hat{V}+\hat{U}\right|\right\rangle
$$

and the kinetic energy (including correlation kinetic energy) of the electrons with spin orientation $s=\uparrow$ or $\downarrow$ is

$$
T_{s}\left(\alpha_{\uparrow}, \alpha_{\downarrow}\right)=\left\langle\alpha_{\uparrow}, \alpha_{\downarrow}\left|\alpha_{s}^{-1} \hat{T}_{s}\right| \alpha_{\uparrow}, \alpha_{\downarrow}\right\rangle .
$$

Neither the external potential $\hat{V}$ nor the bare electronelectron interaction $\hat{U}$ depends on the masses and therefore both $\hat{V}$ and $\hat{U}$ are independent of $\alpha_{\uparrow}$ and $\alpha_{\downarrow}$. Thus by the Feynman-Hellman theorem

$$
\begin{aligned}
\frac{\partial}{\partial \alpha_{s}} E\left(\alpha_{\uparrow}, \alpha_{\downarrow}\right) & =\left\langle\left|\frac{\partial}{\partial \alpha_{s}}\left(\alpha_{\uparrow}^{-1} \hat{T}_{\uparrow}+\alpha_{\downarrow}^{-1} \hat{T}_{\downarrow}+\hat{V}+\hat{U}\right)\right|\right\rangle \\
& =-\alpha_{s}^{-2}\left\langle\left|\hat{T}_{s}\right|\right\rangle=-\alpha_{s}^{-1} T_{s}\left(\alpha_{\uparrow}, \alpha_{\downarrow}\right) .
\end{aligned}
$$

Thus the kinetic energy (KE) of the electrons of spin orientation $s$ in the true system (with $\alpha_{\uparrow}=\alpha_{\downarrow}=1$ ) is

$$
\begin{aligned}
T_{s} & \equiv T_{s}\left(\alpha_{\uparrow}=1, \alpha_{\downarrow}=1\right)=-\left[\alpha_{s} \frac{\partial}{\partial \alpha_{s}} E\left(\alpha_{\uparrow}, \alpha_{\downarrow}\right)\right]_{\alpha_{\uparrow}=\alpha_{\downarrow}=1} \\
& =-\left[\frac{\partial}{\partial \alpha_{s}} E\left(\alpha_{\uparrow}, \alpha_{\downarrow}\right)\right]_{\alpha_{\uparrow}=\alpha_{\downarrow}=1} .
\end{aligned}
$$

This result includes all correlation effects on the kinetic energy, and holds for an arbitrary inhomogeneous manyelectron system under the conditions given earlier.

\section{SPIN-RESOLVED KE VIA DIFFERENTIATION ON MASS: SPIN-POLARIZED ELECTRON GAS}

For the special case of the spin-polarized homogeneous electron gas, the external potential $\hat{V}$ can be taken to represent the effects of two uniform positive backgrounds, one for each spin orientation. Here $\hat{V}$ serves simply to determine the separate densities of the up and down electrons, or equivalently it determines their Fermi wave numbers

$$
k_{F s}=\left(6 \pi^{2} \frac{N_{s}}{V}\right)^{1 / 3},
$$

where $V$ is the fixed volume of the gas and $N_{s}$ is the number of electrons with spin projection $s$. We take the vector potential $A$ to be zero. For this system the Hartree energy is zero. The total ground state energy can thus be broken down into Kohn-Sham kinetic energy and exchange energy for each spin orientation separately, plus the Kohn-Sham correlation energy $E_{c}$, which contains the nontrivial components of the kinetic energy

$$
\begin{gathered}
E=T_{\uparrow}^{(K S)}+E_{\uparrow}^{(X)}+T_{\downarrow}^{(K S)}+E_{\downarrow}^{(X)}+E_{c}, \\
T_{s}^{(K S)}=N_{s} \frac{3}{5} \frac{\hbar^{2} k_{F s}^{2}}{2 m_{s}}, E_{s}^{(X)}=-N_{s} \frac{3 e^{2}}{4 \pi} k_{F s}, \\
E_{c}=N \varepsilon_{c}\left(m_{\uparrow}, k_{F \uparrow} ; m_{\downarrow}, k_{F \downarrow}\right)=N \varepsilon_{c}\left(m \alpha_{\uparrow}, k_{F \uparrow} ; m \alpha_{\downarrow}, k_{F \downarrow}\right),
\end{gathered}
$$

where $m$ is the true electron mass, $N=N_{\uparrow}+N_{\downarrow}$ is the total number of electrons, and $\varepsilon_{c}$ is the correlation energy per particle. Here $k_{F_{s}}$ from (5) is independent of the mass and hence of $\alpha_{s}$. Furthermore the exchange energy does not involve the mass (or $\alpha_{s}$ ), and will therefore not contribute to the kinetic energy in (4). The prescription $-\alpha_{s} \partial / \partial \alpha_{s}$ applied to $T_{s}^{(K S)}=N_{s} \frac{3}{5} \hbar^{2} k_{F_{s}}^{2}\left(2 m \alpha_{s}\right)^{-1}$ just gives $T_{s}^{(K S)}$ back again. Thus (6) and (4) lead to 


$$
T_{s}=T_{s}^{(K S)}-\alpha_{s} \frac{\partial E_{c}}{\partial \alpha_{s}} \equiv T_{s}^{(K S)}+T_{c s} .
$$

where the spin-resolved correlation kinetic energy at the true electron mass is

$$
T_{c s}=-\left.N \frac{\partial \varepsilon_{c}\left(m \alpha_{\uparrow}, k_{F \uparrow} ; m \alpha_{\downarrow}, k_{F \downarrow}\right)}{\partial \alpha_{s}}\right|_{\alpha_{\uparrow}=\alpha_{\downarrow}=1} .
$$

A similar formula holds for the corresponding quantitiy per particle $t_{c s}=T_{c s} / N$ where $N$ is the total number of electrons ignoring spin

$$
t_{c s}=-\left.\frac{\partial \varepsilon^{c}\left(\alpha_{\uparrow} m, k_{F \uparrow} ; \alpha_{\downarrow} m, k_{F \downarrow}\right)}{\partial \alpha_{s}}\right|_{\alpha_{\uparrow}=\alpha_{\downarrow}=1} .
$$

\section{SPIN-RESOLVED PAIR FACTOR AND CORRELATION ENERGY OF THE POLARIZED UNIFORM GAS IN THE STLS SCHEME}

The adiabatic connection formula relates the correlation energy to the spin-resolved pair density $n_{\lambda s s^{\prime}}\left(\vec{r}, \vec{r}^{\prime}\right)$ $=n_{s}(\vec{r}) n_{s^{\prime}}\left(\vec{r}^{\prime}\right) g_{\lambda s s^{\prime}}\left(\vec{r}, \vec{r}^{\prime}\right)$ of a gas with reduced coulomb interaction $\lambda e^{2} /\left|\vec{r}-\vec{r}^{\prime}\right|$ :

$$
\frac{E_{c}}{V}=\frac{1}{2 V} \int_{0}^{1} d \lambda \int \frac{e^{2}}{\left|\vec{r}-\vec{r}^{\prime}\right|} \times \sum_{s s^{\prime}} n_{s}(\vec{r}) n_{s^{\prime}}\left(\vec{r}^{\prime}\right) g_{c \lambda s s^{\prime}}\left(\vec{r}, \vec{r}^{\prime}\right) d \vec{r} d \vec{r}^{\prime} .
$$

Here the pair factor $g$ has been broken down to exhibit the exchange $(x)$ and correlation $(c)$ corrections to the Hartree theory:

$$
g_{\lambda s s^{\prime}}\left(\vec{r}, \vec{r}^{\prime}\right)=1+g_{x \lambda s s^{\prime}}+g_{c \lambda s s^{\prime}} .
$$

For a homogeneous gas (8) can be Fourier transformed to give

$$
\frac{E_{c}}{V}=e^{2} \sum_{s s^{\prime}} n_{s} n_{s^{\prime}} \int_{0}^{1} d \lambda \int_{0}^{\infty} d q g_{c \lambda s s^{\prime}}(q) .
$$

From the fluctuation-dissipation theorem, the pair correlation factor $g_{c \lambda}$ is related to the retarded Kubo density-density response $\chi$ at imaginary frequency

$$
g_{c \lambda s s^{\prime}}(q)=-\frac{1}{n_{s} n_{s^{\prime}}} \frac{\hbar}{\pi} \int_{0}^{\infty}\left[\chi_{\lambda s s^{\prime}}(q, i u)-\delta_{s s^{\prime}} \chi_{0 s s}(q, i u)\right] d u .
$$

Here we evaluate $\chi_{\lambda s s^{\prime}}$ within the STLS scheme, ${ }^{22}$ suitably generalized to resolve the spin components of all quantities. The unique feature of the STLS scheme is the asumption that the perturbed dynamic pair distribution is related to the onebody distribution via the static equilibrium pair factor $g$ :

$$
f_{2 \lambda}\left(\vec{r} \vec{p} s, \vec{r}^{\prime} \vec{p}^{\prime} s^{\prime}, t\right)=f(\vec{r} \vec{p} s t) f\left(\vec{r}^{\prime} \vec{p}^{\prime} s^{\prime} t\right) g_{\lambda s s^{\prime}}\left(\vec{r}, \vec{r}^{\prime}\right) .
$$

After linearization, this form of dynamic response leads to a closed one-body kinetic equation with an effective force, and hence to the RPA-like screening Eqs. (16)-(19) shown later.
The effective pair potential $w$ from this theory satisfies

$$
\vec{\nabla} w_{\lambda s s^{\prime}}\left(\left|\vec{r}-r^{\prime}\right|\right)=g_{s s^{\prime}}\left(\left|\vec{r}-r^{\prime}\right|\right) \vec{\nabla} \frac{e^{2}}{\left|\vec{r}-r^{\prime}\right|},
$$

or in Fourier space

$$
\begin{aligned}
w_{\lambda s s^{\prime}}(q)= & \frac{4 \pi \lambda e^{2}}{q^{2}}+\frac{\lambda e^{2}}{2 \pi q^{3}} \times \int_{0}^{\infty} q^{\prime}\left[2 q q^{\prime}\right. \\
& \left.+\left(q^{2}-q^{\prime 2}\right) \ln \left|\frac{q+q^{\prime}}{q-q^{\prime}}\right|\right] \Delta g_{\lambda s s^{\prime}}\left(q^{\prime}\right) d q^{\prime} .
\end{aligned}
$$

Here

$$
\begin{gathered}
g_{\lambda s s^{\prime}}(q)=(2 \pi)^{3} \delta^{3}(\vec{q})+\Delta g_{\lambda s s^{\prime}}(q), \\
\Delta g_{\lambda s s^{\prime}}(q)=g_{s s^{\prime}}^{\operatorname{exch}}(q)+g_{c \lambda s s^{\prime}}(q), \\
g_{s s^{\prime}}^{\text {exch }}(q)=-\delta_{s s^{\prime}} n_{0 s}^{-1}\left[1-\frac{3}{4} x+\frac{1}{16} x^{3}\right], x=q / k_{F s^{\prime}} .
\end{gathered}
$$

With the arguments $q$ and $i u$ omitted for simplicity, the spin-resolved STLS screening equations can be written in RPA-like fashion in terms of the bare response $\chi_{0 s s^{\prime}}$ and the effective pair potential $w_{\lambda s s^{\prime}}$. The spin-resolved linear density perturbation satisfies

$$
\delta n_{s}=\chi_{0 s}\left(\delta V_{s}^{\mathrm{ext}}+\sum_{s^{\prime}} w_{s s^{\prime} \lambda} \delta n_{s^{\prime}}\right) .
$$

The solution of these two equations can be written

$$
\delta n_{s}=\sum_{s^{\prime}} \chi_{\lambda s s^{\prime}} \delta V_{s^{\prime}}^{\mathrm{ext}}
$$

where the interacting response is

$$
\begin{aligned}
& \chi_{\lambda \uparrow \uparrow}=D_{\lambda}^{-1}\left(1-\chi_{0 \downarrow \downarrow} w_{\lambda \downarrow \downarrow}\right) \chi_{0 \uparrow \uparrow}, \\
& \chi_{\lambda \uparrow \downarrow}=D_{\lambda}^{-1} \chi_{0 \downarrow \downarrow} w_{\lambda \uparrow \downarrow} \chi_{0 \uparrow \uparrow}=\chi_{\lambda \downarrow \uparrow}, \\
& \chi_{\lambda \downarrow \downarrow}=D_{\lambda}^{-1}\left(1-\chi_{0 \uparrow \uparrow} w_{\lambda \uparrow \uparrow}\right) \chi_{0 \downarrow \downarrow} .
\end{aligned}
$$

Here

$$
D=\left(1-\chi_{0 \downarrow \downarrow} w_{\downarrow \downarrow}\right)\left(1-\chi_{0 \uparrow \uparrow} w_{\uparrow \uparrow}\right)-\chi_{0 \downarrow \downarrow} w_{\downarrow \uparrow} \chi_{0 \uparrow \uparrow} w_{\uparrow \downarrow} .
$$

The bare response is

$$
\begin{gathered}
\chi_{0 s s}\left(k_{F s}, q, i u\right)=-\frac{m_{s} k_{F s}}{4 \pi^{2} \hbar^{2}} \chi_{00}\left(Q_{s}, \gamma_{s}\right), Q_{s}=\frac{q}{2 k_{F s}}, \\
\gamma_{s}=\frac{\hbar u /\left(4 E_{F s}\right)}{Q_{s}}=\frac{u}{v_{F s} q}=m_{s} \frac{u}{\hbar k_{F s} q}, \\
\chi_{00}(Q, \gamma)=1+\frac{1+\gamma^{2}-Q^{2}}{4 Q} \ln \frac{(1+Q)^{2}+\gamma^{2}}{(1-Q)^{2}+\gamma^{2}} \\
-\gamma \arctan \left(\frac{2 \gamma}{Q^{2}+\gamma^{2}-1}\right),
\end{gathered}
$$


TABLE I. Spin-resolved KE per electron $t_{c s}=T_{c s} / N$ (Hartree): STLS data from present work.

\begin{tabular}{ccccccccccc}
\hline \hline$\zeta$ & $t_{c \uparrow}$ & $t_{c \downarrow}$ & $t_{c \uparrow}$ & $t_{c \downarrow}$ & $t_{c \uparrow}$ & $t_{c \downarrow}$ & $t_{c \uparrow}$ & $t_{c \downarrow}$ & $t_{c \uparrow}$ & $t_{c \downarrow}$ \\
\hline & $r_{s}=1$ & $r_{s}=1$ & $r_{s}=2$ & $r_{s}=2$ & $r_{s}=3$ & $r_{s}=3$ & $r_{s}=4$ & $r_{s}=4$ & $r_{s}=5$ & $r_{s}=5$ \\
1 & 0.02154 & 0 & 0.01443 & 0 & 0.01091 & 0 & 0.008766 & 0 & 0.007279 & 0 \\
0.8 & 0.02361 & 0.004889 & 0.01541 & 0.003169 & 0.01150 & 0.002372 & 0.009114 & 0.001858 & 0.007551 & 0.001569 \\
0.6 & 0.023602 & 0.00902 & 0.01534 & 0.00584 & 0.01141 & 0.004349 & 0.009014 & 0.003421 & 0.007452 & 0.002851 \\
0.4 & 0.02259 & 0.01266 & 0.01466 & 0.008203 & 0.01089 & 0.006098 & 0.008599 & 0.004803 & 0.007104 & 0.003985 \\
0.2 & 0.02089 & 0.01587 & 0.01355 & 0.01028 & 0.01006 & 0.007639 & 0.007937 & 0.006021 & 0.006558 & 0.004984 \\
0.0 & 0.01862 & 0.01862 & 0.01207 & 0.01207 & 0.008966 & 0.008966 & 0.00707 & 0.00707 & 0.005845 & 0.005845 \\
\hline \hline
\end{tabular}

$$
k_{F s}=\left(6 \pi^{2} n_{s}\right)^{1 / 3} .
$$

For specified values of the spin densities $n_{\uparrow}, n_{\downarrow}$ and masses $m_{\uparrow}=\alpha_{\uparrow} m, m_{\downarrow}=\alpha_{\downarrow} m$, Eqs. (10)-(21) form a self-consistent loop that we solved numerically, starting from an exchangeonly pair distribution and continuing iterations till the pair distributions and energy [from (9)] were converged to the desired accuracy. We also evaluated the pure RPA energies.

\section{IMPLEMENTATION OF THE MASS-DIFFERENTIATION KE FORMULA WITHIN SPIN-RESOLVED STLS}

Equation (7) requires differentiation with respect to the mass factors $\alpha_{s}$. The dependence of the spin-STLS correlation energy on the masses $m_{\uparrow}, m_{\downarrow}$ is solely via the mass dependences of the bare susceptibilty exhibited in Eq. (20). The required mass differentiations might therefore in principle be carried through analytically but this is complicated by the self-consistency of the equations. We found it simpler to evaluate $\varepsilon_{c}$ for a set of $\alpha_{s}$ values near to unity, and to use numerical differentiation. With a five-point differentiation formula and various spacings we obtained consistent values $t_{c \uparrow}$ and $t_{c \downarrow}$ at the $0.1 \%$ level, sufficient for present purposes.

Table I shows $t_{c \uparrow}$ and $t_{c \downarrow}$ as functions of $r_{s}$ and the spin polarization $\zeta=\left(n_{\uparrow}-n_{\downarrow}\right) / n$.

Qian and Vignale ${ }^{24}$ postulated [their Eq. (29)] that, at fixed $r_{s}, t_{c \uparrow}-t_{c \downarrow}$ is a linear fuction of the spin polarization $\zeta$. To investigate this, in Fig. 1 we plot, for each fixed $r_{s}$ value, the variable $y=\left[t_{c \uparrow}\left(r_{s}, \zeta\right)-t_{c \downarrow}\left(r_{s}, \zeta\right)\right] / t_{c}\left(r_{s}, \zeta=1\right)$ as a function of spin polarization $\zeta$. Within the STLS theory, the resulting curves (symbols) are roughly linear, $y \approx \zeta$, but there are noticeable departures from linearity that are different for different $r_{s}$ values. The curves are somewhat more linear and less $r_{s}$-dependent within the pure RPA theory (solid and dashed lines).

Figure 2 shows a slightly different but remarkably accurate scaling exhibited by the STLS data for the spin components of the correlation kinetic energy. Consider the spinasymmetry $x$ of the correlation kinetic energy, defined by

$$
x \equiv\left[t_{c \uparrow}\left(r_{s}, \zeta\right)-t_{c \downarrow}\left(r_{s}, \zeta\right)\right] / t_{c}\left(r_{s}, \zeta\right) .
$$

When $x$ is plotted versus the polarization $\zeta=\left(n_{\uparrow}-n_{\downarrow}\right) / n$, we find empirically that the resulting curve is closely the same for all $r_{s}$ values in the metallic range (symbols in Fig. 2). This scaling is manifested by the three symbols being essentially coincident at each data point. This scaling is not nearly so well obeyed within the RPA approximation (solid and dashed lines). It would be interesting to see if diffusion Monte Carlo (DMC) results agree with the STLS scaling.

Noting that $x$ is roughly linear and, by its definition, must vanish at $\zeta=0$ and equal 1 at $\zeta=1$, we can model the universal STLS curve as follows:

$$
x(\zeta)=\zeta-a \zeta^{p}\left(1-\zeta^{q}\right) \text { independent of } r_{s} .
$$

By optimizing the fit to our numerical curves (see the Appendix) we found $a=0.27117, p=1.0000, q=4.75$. The excellent fit thus obtained is illustrated in Fig. 2 (thin solid curve).

From (23) (plus $t_{c \uparrow}+t_{c \downarrow}=t_{c}$ ) we have

$$
\begin{aligned}
& t_{c \uparrow}\left(r_{s}, \zeta\right)=\frac{1+x(\zeta)}{2} t_{c}\left(r_{s}, \zeta\right), \\
& t_{c \downarrow}\left(r_{s}, \zeta\right)=\frac{1-x(\zeta)}{2} t_{c}\left(r_{s}, \zeta\right),
\end{aligned}
$$

where from (24)

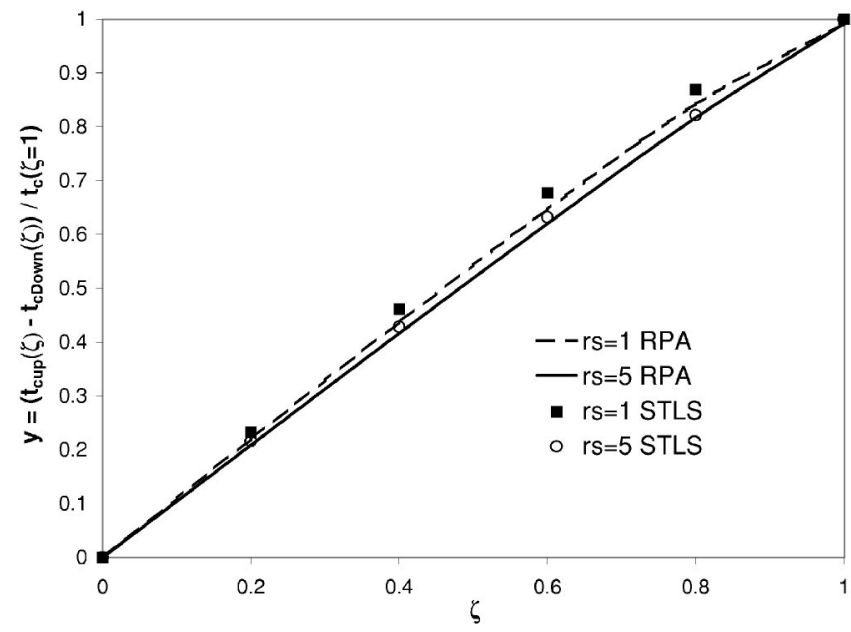

FIG. 1. $y \equiv\left[t_{c \uparrow}\left(r_{s}, \zeta\right)-t_{c \downarrow}\left(r_{s}, \zeta\right)\right] / t_{c}\left(r_{s}, \zeta=1\right)$ as a function of spin polarization $\zeta=\left(n_{\uparrow}-n_{\downarrow}\right) /\left(n_{\uparrow}-n_{\downarrow}\right)$ for the three-dimensional homogeneous electron gas. Filled squares: STLS calculation for $r_{s}=1$. Open circles: STLS calculation for $r_{s}=5$. Dashed line: RPA calculation for $r_{s}=1$. Continuous line: RPA calculation for $r_{s}=5$. 


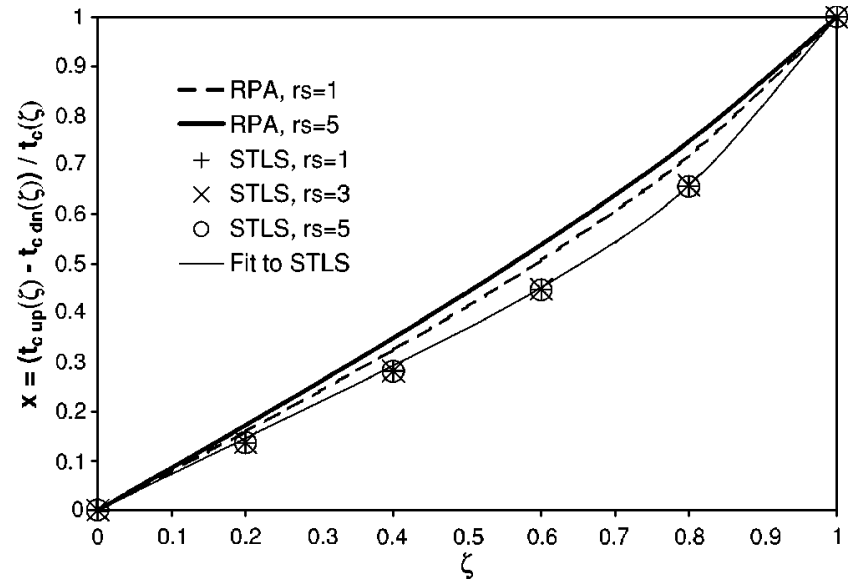

FIG. 2. The spin asymmetry $x \equiv\left[t_{c \uparrow}\left(r_{s}, \zeta\right)-t_{c \downarrow}\left(r_{s}, \zeta\right)\right] / t_{c}\left(r_{s}, \zeta\right)$ of the kinetic correlation energy as a function of polarization $\zeta$. Symbols: STLS calculations with $r_{s}=1,3,5$. Thin solid line: $r_{s}$-independent fit to the STLS calculations from Eq. (27). Dashed line: RPA calculation for $r_{s}=1$. Thick solid line: RPA calculation for $r_{s}=5$.

$$
x(\zeta)=\zeta-0.27117 \zeta\left(1-\zeta^{4.75}\right) \text { independent of } r_{s} .
$$

Thus we have the spin-resolved correlation KE in terms of the corresponding spin-unresolved quantity $t_{c}\left(r_{s}, \zeta\right)$.

Perdew and Wang [see Eq. (A9) of "PW92"] found that

$$
\begin{aligned}
t_{c}\left(r_{s}, \zeta\right) & =-4 \varepsilon_{c}\left(r_{s}, \zeta\right)+3\left(\frac{1+\zeta}{2} \mu_{c}^{\uparrow}\left(r_{s}, \zeta\right)+\frac{1-\zeta}{2} \mu_{c}^{\downarrow}\left(r_{s}, \zeta\right)\right) \\
& =-\frac{\partial}{\partial r_{s}}\left[r_{s} \varepsilon_{c}\left(r_{s}, \zeta\right)\right]
\end{aligned}
$$

where $\varepsilon_{c}\left(r_{s}, \zeta\right)$ is the spin-unresolved correlation energy of the partially spin-polarized gas. In (28):

$$
\mu_{c}^{\uparrow}\left(r_{s}, \zeta\right)=\left.\frac{\partial\left(n \varepsilon_{c}\right)}{\partial n_{\uparrow}}\right|_{n_{\downarrow}}
$$

is the Kohn-Sham potential of spin-up electrons, and similarly for $\mu_{c}^{\downarrow}\left(r_{s}, \zeta\right)$. PW92 provided analytic fits for $\varepsilon_{c}\left(r_{s}, \zeta\right)$, $\mu_{c}^{\uparrow}\left(r_{s}, \zeta\right)$, and $\mu_{c}^{\downarrow}\left(r_{s}, \zeta\right)$ based on interpolation/extrapolation of DMC data. These PW92 fits are available in the widelydistributed PBE code,${ }^{10}$ if one chooses the density gradients to be zero. It is therefore probably more accurate and convenient to use the first equality in (28) rather than numerically performing the $r_{s}$ differentiation indicated in the second equality. One could probably produce fits of the same general form for our STLS energy data, but since the STLS total energy data are rather similar to the DMC data (see later) we suggest using the existing PW92 fits to $\varepsilon_{c}^{\mathrm{DMC}}, \mu_{c}^{\uparrow}\left(r_{s}, \zeta\right)$, and $\mu_{c}^{\downarrow}\left(r_{s}, \zeta\right)$ as just described.

Equations (23)-(28) provide a convenient near-analytic way to predict the up- and down-spin components of the correlation kinetic energy for spin-polarized gases in the metallic density range, and they constitute the principal practical result of the present work.

\section{COMPARISON WITH A PROPOSED SCHEME BASED ON DIFFERENTIATION OF CORRELATION ENERGY WITH RESPECT TO WIGNER-SEITZ RADIUS}

Starting from an early suggestion by Caccamo et al., ${ }^{25}$ Gori-Giorgi and Perdew ${ }^{20}$ proposed that the spin-resolved components of the correlation kinetic energy might be given at least approximately by

$$
t_{c s}=-\frac{\partial}{\partial r_{s}}\left(r_{s} \varepsilon_{c s}\right),
$$

where $\varepsilon_{c s}$ is the correlation energy per electron of spin $s$. The corresponding formula $t_{c}=-\partial\left(r_{s} \varepsilon_{c}\right) / \partial r_{s}$ for the spin unresolved correlation $\mathrm{KE}$ is exact and can be proven by use of the Virial Theorem: see, e.g., Eqs. (23)-(24) of Kwon et al. ${ }^{26}$ and also Eq. (28) of the present work. The use of the formula (30) for the spin-resolved KE is, however, not directly sanctioned by the Virial theorem, and indeed there are doubts about it, as surmised by Gori-Giorgi and Perdew and mentioned already in the Introduction earlier. Gori-Giorgi and Perdew used (30) to obtain a prediction for the difference $t_{c \uparrow}-t_{c \downarrow}$ as a function of spin polarization $\zeta$. Figure 3 shows the spin-KE-polarization quantity $x$ defined in Eq. (23) as a function of spin polarization $\zeta$, evaluated via three different

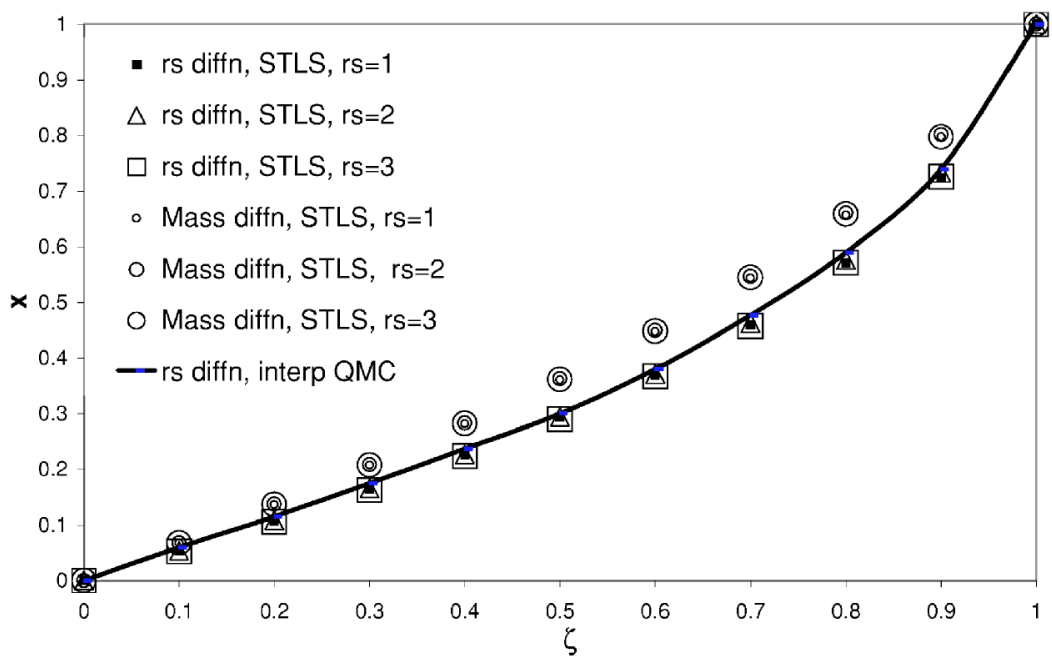

FIG. 3. Approximations for the spin asymmetry $x$ of the kinetic correlation energy $t_{c}$ as defined in Eq. ${ }^{23}$ Thick line: Eq. (30) evaluated by Gori-Giorgi and Perdew ${ }^{27}$ using interpolated QMC data. Squares and triangles: evaluation of Eq. (30) using our STLS data for $r_{s}=1,2,3$. Circles: the exact Eq. (7) using STLS data for $r_{s}=1,2,3$. 


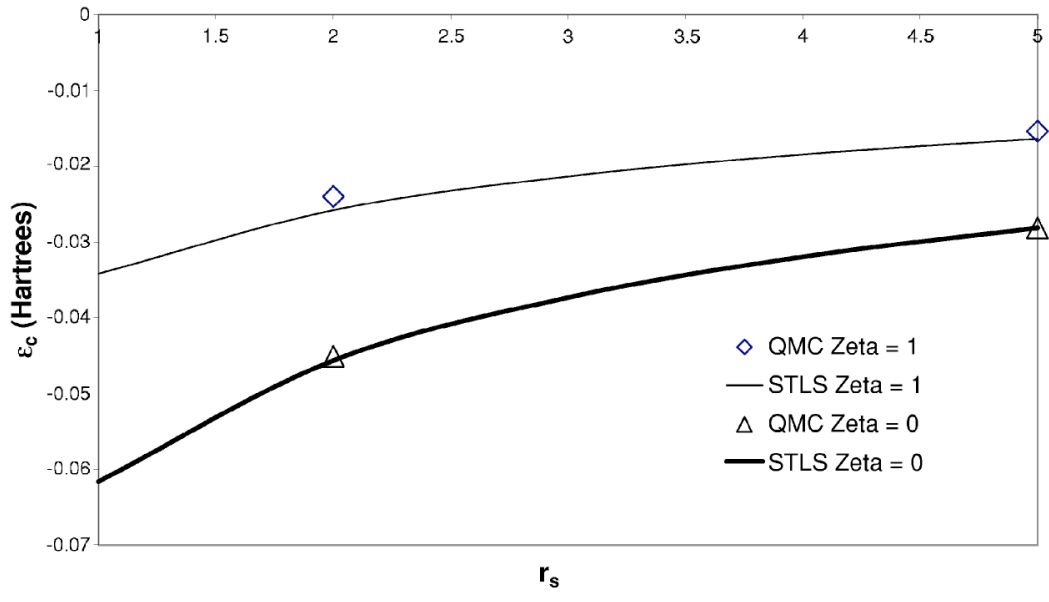

FIG. 4. Correlation energy per electron of the homogeneous electron gas for the unpolarized $(\zeta=0)$ and fully polarized $(\zeta=1)$ cases. Thick line: STLS calculation, $\zeta=0$. Thin line: STLS calculation, $\zeta=1$. Open triangles: DMC calculation, ${ }^{4}$ $\zeta=0$. Open diamonds: DMC calculation, ${ }^{4} \zeta=1$. prescriptions. The thick lines represent calculations of $x$ by Gori-Giorgi and Perdew ${ }^{27}$ based on Eq. (30) using interpolated QMC data. This curve has been averaged over data for $r_{s}=1,3,5,10$. (The results of Gori-Giorgi and Perdew varied little with $r_{s}$ in this range.) In Fig. 3 the triangles and squares represent the semiempirical formula (30) evaluated this time by us, using spin-resolved STLS for $\varepsilon_{c s}$. The circles in Fig. 3 represent a calculation via our exact mass-differentiation formula (7), evaluated by us using the spin-resolved STLS formalism. The coincidence of the symbols confirms that the quantity $x$ is almost independent of $r_{s}$ in the metallic range.

Figure 3 shows that:

(a) The use of the STLS approximation instead of QMC data for $\varepsilon_{c s}$ makes little change to the values of $x$ calculated from Eq. (30). This is a partial confirmation of the suitability of STLS for predicting the spin breakdown of the correlation kinetic energy; and

(b) The use of the semiempirical formula (30) instead of the exact mass-differentiation formula (7) does make a noticeable difference to the values of $x$. Clearly the exact formula should be preferred.

\section{HOW ACCURATE IS STLS FOR THE SPIN- RESOLVED ENERGETICS?}

The STLS method used here is based on the bold assumption (11) about the factorization of the dynamic pair distribution, and furthermore does not satisfy the Kimball coulomb cusp condition. Nevertheless it is well-known to give excellent correlation energies for the spin-unpolarized electron gas at metallic densities and even up to $r_{s}=40$. Can it be expected to give similar accuracy for spin-resolved quantities? Some evidence is available by looking at the total correlation energy $\varepsilon_{c}\left(r_{s}, \zeta=0\right), \varepsilon_{c}\left(r_{s}, \zeta=1\right)$ of the unpolarized and fully polarized gases, for which QMC data is available. ${ }^{4}$ This is shown in Fig. 4. The agreement between STLS and QMC is still good at $\zeta=1$, though not quite as good as at $\zeta$ $=0$.

Furthermore, although we have concluded that the quantity (30) is not in general an accurate representation of $t_{c s}$, our STLS evaluation of the right-hand side of (30) agrees well with the DMC-based interpolation of the same quantity given by Gori-Giorgi and Perdew. ${ }^{27}$ This again enhances confidence in the use of STLS for spin-resolved quantities. Ultimately, though, the spin-resolved quantities discussed here should be evaluated directly within a more accurate method such as diffusion Monte Carlo, in order to test the validity of the surprising scaling discovered here within the STLS approximation.

\section{OTHER POSSIBLE APPROACHES}

Other approaches may be possible for evaluation of the spin-resolved KE. For example, a spin-resolved Virial theorem has been given by Isihara. ${ }^{28}$ Its use requires evaluation of the expectation of a modified form $X$ of the electronelectron interaction operator

$$
X=\left(\left|\vec{r}_{1}\right|^{2}-\left|\vec{r}_{2}\right|^{2}\right) /\left|\vec{r}_{1}-\vec{r}_{2}\right|^{3}
$$

The Isihara approach was used ${ }^{28}$ to obtain the spin-resolved correlation $\mathrm{KE} T_{s}^{(c)}$ for atomic situations. This approach might be applicable to electron gases also. There is a potential problem, however, in that the definition (31) appears to depend on the choice of spatial origin, not a convenient property when treating the homogeneous gas. Our method is straightforward and has no such caveats for the problem at hand.

$\mathrm{Nagy}^{29}$ has related the spin-virial approach to the KohnSham KE but not to the correlation part of the KE studied here.

Magyar et $a l .{ }^{30}$ have discussed spin scaling relations and a spin-dependent adiabatic connection formalism. Their work related the xc energy of the actual system to that of a fictitious system having one spin density, say $n_{\uparrow}$, replaced by zero. Correspondingly they gave an adiabatic connection formula for the quantity

$$
\Delta E_{x c}\left[n_{\uparrow}, n_{\downarrow}\right]=E_{x c}\left[n_{\uparrow}, n_{\downarrow}\right]-E_{x c}\left[0, n_{\downarrow}\right] .
$$

This introduces a type of spin-dependent decomposition, because Eq. (32) gives a different connection according which spin species, $\uparrow$ or $\downarrow$, has its density reduced to zero. It is not clear how this relates to the quantity $T_{s}$ [our Eq. (3) with $\alpha_{\uparrow}=\alpha_{\downarrow}=1$ ], which gives the spin-resolved KE when both spin species are present. $T_{s}$ occurs naturally when the 
third moment sum rule is invoked in a spin context, which is the reason for the focus upon $T_{s}$ in the present work.

\section{SUMMARY AND DISCUSSION}

We have introduced an unequivocal prescription [Eq. (4)] for finding the spin-resolved kinetic energy of an interacting many-electron system, and have applied it to obtain a massdifferentiation formula (7) for the spin-resolved correlation kinetic energy $t_{c s}\left(r_{s}, \zeta\right)$ per electron of the spin-polarized homogeneous three-dimensional electron gas. $t_{c s}$ is a a quantity required for use in the spin current density theory of inhomogeneous spin-polarized electronic systems. ${ }^{24}$ That theory in turn is expected to be important in the analysis of nanoscale spintronic devices.

Using a spin generalization of the STLS ${ }^{22}$ theory, we have implemented our prescription (7) to obtain $t_{c \uparrow}$ and $t_{c \downarrow}$ for the homogeneous three-dimensional electron gas at metallic densities $\left(1<r_{s}<5\right)$ and at all spin polarizations $(0 \leqslant \zeta \leqslant 1)$. (See Table I). We have found that the STLS data obey a remarkably accurate scaling relation: when the "spin asymmetry of the correlation KE," defined by $x \equiv\left(t_{c \uparrow}-t_{c \downarrow}\right) /\left(t_{c \uparrow}\right.$ $\left.+t_{c \downarrow}\right)$, is plotted against spin polarization $\zeta \equiv\left(n_{\uparrow}-n_{\downarrow}\right) /\left(n_{\uparrow}\right.$ $\left.+n_{\downarrow}\right)$, the result is accurately independent of the density parameter $r_{s}$ across the metallic range (see symbols in Fig. 2). This scaled behavior is parametrized to a percent or so by the analytic formula (27) (thin solid line in Fig. 2), which is a principal result of the present work. An identical calculation done within the random phase approximation does not yield the same results, nor are the RPA numbers nearly as independent of $r_{s}$. Using our scaling relation (27) plus the exact formulae (25), (26), and (28), one can use any accurate tabulation or parametrization of the spin-unresolved correlation energy $\varepsilon_{c}\left(r_{s}, \zeta\right)$ and spin correlation potentials $\mu_{c}^{\uparrow}, \mu_{c}^{\downarrow}$ to predict spin-resolved KE quantities of the spin-polarized gas. For example, the parametrizations given in $\mathrm{PW} 92^{9}$ should be suitable.

It will be interesting to see whether the remarkable $r_{s}$ independence of the quantity $x\left(r_{s}, \zeta\right)$, identified here in Eq. (23) and Fig. 2, survives when all quantities are evaluated in a more accurate theory such as diffusion Monte Carlo, rather than in the STLS approximation used here.

\section{ACKNOWLEDGMENTS}

The authors thank J. Perdew and P. Gori-Giorgi for useful communications and data. J.F.D. acknowledges support from an Australian Research Council Large Grant. G.V. acknowledges NSF Grant No. DMR-0313681. Computer time was provided by Research Computing Services at Griffith University.

\section{APPENDIX: FITTING $\boldsymbol{A}, \boldsymbol{P}, \mathrm{AND} Q$}

The derivative of (24) is

$$
\frac{d x}{d \zeta}=1-a p \zeta^{p-1}\left(1-\zeta^{q}\right)+q a \zeta^{p} \zeta^{q-1} .
$$

Thus

$$
\left.\frac{d x}{d \zeta}\right|_{\zeta=0}=1-a p 0^{p-1} \text { assuming } p+q-1>0 .
$$

Inspection of the data shows that the initial slope $d x /\left.d \zeta\right|_{\zeta=0}$ is finite but not equal to 1 . Thus $0^{p-1}$ must be finite (neither zero nor infinity), and so we require $p=1$. Thus our fit becomes

$$
\begin{gathered}
x(\zeta)=\zeta-a \zeta\left(1-\zeta^{q}\right), \\
\frac{d x}{d \zeta}=1-a\left(1-\zeta^{q}\right)+q a \zeta^{q}=1-a\left(1+(q-1) \zeta^{q}\right) .
\end{gathered}
$$

It is convenient to work with the difference (deviation) of $x$ below the straight line $x=\zeta$. Thus we define a positive difference

$$
\Delta=\zeta-x(\zeta)=a \zeta\left(1-\zeta^{q}\right) .
$$

We will choose $q$ and $a$ to fit the size of the maximum deviation $\Delta_{\max }$ and the position $\zeta_{0}$ where it occurs. To implement this we note that the derivative of the difference function is

$$
\frac{d \Delta}{d \zeta}=a\left[1-(q+1) \zeta^{q}\right] .
$$

This is zero [implying maximum deviation of $x(\zeta)$ from the straight line $x=\zeta$ ] at a point $\zeta_{0}$ such that

$$
1=(q+1) \zeta_{0}^{q} \text {. }
$$

The max deviation point $\zeta_{0}$ was found, from the STLS data for $r_{s}=3$, to be $\zeta_{0}=0.69_{2}$. (A4) has the trivial solution $q=0$ but is there another? By tabulation we found that $q=4.75$ satisfies (A4) with the correct $\zeta_{0}$. The maximum deviation of the STLS data is $\Delta_{\max }=0.155$. Thus, from (A4), $a$ is determined by

$$
\begin{gathered}
\Delta_{\max }=a \zeta_{0}\left(1-\zeta_{0}^{q}\right) \\
a=\Delta_{\max } \zeta_{0}^{-1}\left(1-\zeta_{0}^{q}\right)^{-1}=0.155(0.692)^{-1}\left(1-0.692^{4.75}\right)^{-1} \\
=0.27117 .
\end{gathered}
$$

This leads to the following fit:

$$
x(\zeta)=\zeta-0.27117 \zeta\left(1-\zeta^{4.75}\right) .
$$


${ }^{1}$ P. Nozières and D. Pines, Phys. Rev. 111, 442 (1958).

${ }^{2}$ M. Gell-Mann and K. A. Brueckner, Phys. Rev. 106, 364 (1957).

${ }^{3}$ M. Gell-Mann, Phys. Rev. 106, 369 (1957).

${ }^{4}$ D. M. Ceperley and B. J. Alder, Phys. Rev. Lett. 45, 566 (1980).

${ }^{5}$ B. Tanatar and D. M. Ceperley, Phys. Rev. B 39, 5005 (1989).

${ }^{6}$ F. Rapisarda and G. Senatore, Aust. J. Phys. 49, 161 (1996).

${ }^{7}$ C. Attaccalite, S. Moroni, P. Gori-Giorgi, and G. Bachelet, Phys. Rev. Lett. 88, 256601 (2002).

${ }^{8}$ P. Hohenberg and W. Kohn, Phys. Rev. 136, B864 (1964).

${ }^{9}$ J. P. Perdew and Y. Wang, Phys. Rev. B 45, 13244 (1992).

${ }^{10}$ J. P. Perdew, K. Burke, and M. Ernzerhof, Phys. Rev. Lett. 77, 3865 (1996).

${ }^{11}$ E. K. U. Gross and W. Kohn, Adv. Quantum Chem. 21, 255 (1990).

${ }^{12} \mathrm{H}$. Ohno, Science 281, 951 (1998).

${ }^{13}$ Semiconductor Spintronics and Quantum Computation, edited by D. Awshalom, N. Samarth, and D. Loss (Springer-Verlag, Berlin, 2001).

${ }^{14}$ A. Wolf, Science 294, 1488 (2001).

${ }^{15}$ Z. Qian and G. Vignale, Phys. Rev. Lett. 88, 056404 (2002).

${ }^{16}$ L. D. Landau, E. M. Lifshitz, and L. P. Pitaevski, Statistical Physics, Course of Theoretical Physics Vol. 9 (Reed, Oxford, 1980).
${ }^{17}$ B. Goodman and A. Sjolander, Phys. Rev. B 8, 200 (1973).

${ }^{18}$ Z. Qian, A. Constantinescu, and G. Vignale, Phys. Rev. Lett. 90, 066402 (2003).

${ }^{19}$ Z. Qian and G. Vignale, Phys. Rev. B 68, 195113 (2003).

${ }^{20}$ P. Gori-Giorgi and J. P. Perdew, Phys. Rev. B 69, 041103(R) (2004).

${ }^{21}$ M. Boninsegni and D. M. Ceperley, Phys. Rev. Lett. 74, 2288 (1995).

${ }^{22}$ K. S. Singwi, M. P. Tosi, R. H. Land, and A. Sjolander, Phys. Rev. 176, 589 (1968).

${ }^{23}$ P. Vashishta, P. Bhattacharyya, and K. S. Singwi, Phys. Rev. B 10, 5108 (1974).

${ }^{24}$ Z. Qian and G. Vignale, Phys. Rev. Lett. 88, 056404 (2002).

${ }^{25}$ C. Caccamo, G. Pizzimenti, and M. P. Tosi, Nuovo Cimento Soc. Ital. Fis., B 31B, 53 (1976).

${ }^{26}$ Y. Kwon, D. M. Ceperley, and R. M. Martin, Phys. Rev. B 48, 12037 (1993).

${ }^{27}$ P. Gori-Giorgi and J. P. Perdew (private communication).

${ }^{28}$ M. Isihara, Bull. Chem. Soc. Jpn. 58, 2472 (1985).

${ }^{29}$ A. Nagy, Int. J. Quantum Chem. 49, 353 (1994).

${ }^{30}$ R. J. Magyar, T. K. Whittingham, and K. Burke, Phys. Rev. A 66, 022105 (2002). 